\title{
Simvastatin prevents large blood pressure variability induced aggravation of cardiac hypertrophy in hypertensive rats by inhibiting RhoA/Ras-ERK pathways
}

\author{
Narimasa Takayama ${ }^{1}$, Hisashi Kai ${ }^{1}$, Hiroshi Kudo ${ }^{1}$, Suguru Yasuoka ${ }^{1}$, Takahiro Mori ${ }^{1}$, Takahiro Anegawa ${ }^{1}$, \\ Mitsuhisa Koga ${ }^{2}$, Hidemi Kajimoto ${ }^{2}$, Yoshitaka Hirooka ${ }^{3}$ and Tsutomu Imaizumi ${ }^{1}$
}

Pronounced variability in blood pressure (BP) is an aggravating factor of hypertensive end-organ damage. However, its pathogenesis remains unknown. Statins have various protective effects on the cardiovascular system. Thus, we determined whether simvastatin would attenuate the aggravation of hypertensive cardiac remodeling in a rat model of hypertension with large BP variability, and also investigated the signaling mechanism involved in its effect. A model of hypertension with large BP variability was created by performing bilateral sinoaortic denervation (SAD) in spontaneously hypertensive rats (SHRs). A SAD or sham operation was performed in 12 -week-old rats. Thereafter, simvastatin $\left(0.2 \mathrm{mg} \mathrm{kg}^{-1}\right.$ per day) or vehicle was intraperitoneally administered every day. After 6 weeks, telemetric recordings revealed that SAD enhanced BP variability without changing the mean BP. SAD increased myocyte hypertrophy, myocardial fibrosis and macrophage infiltration associated with the upregulation of brain natriuretic peptide (BNP), type I procollagen, transforming growth factor (TGF)- $\beta$ and monocyte chemoattractant protein (MCP)-1, and activation of RhoA, Ras and ERK1/2. Simvastatin did not change the mean BP or BP variability in SAD-operated SHRs. In SAD-operated SHRs, simvastatin attenuated myocyte hypertrophy and BNP expression, as well as RhoA, Ras and ERK1/2 activities. In contrast, simvastatin did not change myocardial fibrosis, macrophage infiltration, or the expression of procollagen and TGF- $\beta$ or MCP-1 in SAD-operated SHRs. Simvastatin did not affect serum lipid levels. In conclusion, simvastatin attenuated the large BP variability-induced aggravation of cardiac hypertrophy, but not myocardial fibrosis, in SHRs. The activation of RhoA/Ras-ERK pathways may contribute to the aggravation of cardiac hypertrophy by a combination of hypertension and large BP variability.

Hypertension Research (2011) 34, 341-347; doi:10.1038/hr.2010.229; published online 2 December 2010

Keywords: blood pressure variability; cardiac hypertrophy; signaling; statin

\section{INTRODUCTION}

Along with diurnal blood pressure (BP) changes, ${ }^{1,2}$ an increase in short-term BP variability is a characteristic feature of hypertension, especially in the elderly and in patients with carotid atherosclerosis. ${ }^{3-6}$ Large BP variability aggravates cardiac remodeling in hypertensive patients. ${ }^{7-9}$ Further, large BP variability is an independent risk factor for cardiovascular events in hypertensive patients. ${ }^{10-12}$ However, the mechanism by which hypertensive cardiac remodeling is aggravated by increased BP variability remains unknown. Recently, we have created a rat model exhibiting a combination of hypertension and large $\mathrm{BP}$ variability by performing bilateral sinoaortic denervation (SAD) in spontaneously hypertensive rats (SHRs). ${ }^{13}$ This model can also be considered to represent the characteristic features of hypertension in the elderly or those having carotid atherosclerosis. ${ }^{14}$ We have shown that SAD induces cardiac inflammation, which leads to aggravation of hypertensive remodeling (myocyte hypertrophy and myocardial fibrosis) in SHRs. ${ }^{13}$ It is thought that cardiac remodeling in this model would reflect the synergistic effects of the response to sustained pressure overload and the response to increased BP variability.

Statins, or 3-hydroxy-3-methylglutaryl-coenzyme A (HMG-CoA) reductase inhibitors, have various protective effects on the cardiovascular system independent of their lipid-lowering effects. ${ }^{15,16}$ The targets of statins include the small GTP-binding proteins RhoA and Ras. Increased mechanical stress is one of the triggers that activate the RhoA and Ras pathways in cardiac myocytes. ${ }^{17}$

${ }^{1}$ Department of Internal Medicine, Division of Cardio-Vascular Medicine, Kurume University School of Medicine, Kurume, Japan; ${ }^{2}$ Cardiovascular Research Institute, Kurume University School of Medicine, Kurume, Japan and ${ }^{3}$ Cardiovascular Medicine, Kyushu University Graduate School of Medical Sciences, Fukuoka, Japan

Correspondence: Dr H Kai, Department of Internal Medicine, Division of Cardio-Vascular Medicine, Kurume University School of Medicine, 67 Asahimachi, Kurume 830-0011, Japan.

E-mail: naikai@med.kurume-u.ac.jp

Received 15 June 2010; revised 26 August 2010; accepted 9 September 2010; published online 2 December 2010 
Accordingly, we hypothesized that statins may prevent cardiac hypertrophy in response to dynamic, short-term BP changes along with hypertrophy induced by static, chronic BP elevation. Statins can attenuate hypertensive hypertrophy in animal models. ${ }^{18,19}$ The aims of this study were to investigate the effect of simvastatin on the large BP variability-induced aggravation of hypertensive cardiac remodeling in SHRs, and to determine the signaling mechanism involved in such an effect.

\section{METHODS}

The study protocol was reviewed and approved by the Animal Care and Treatment Committee of Kurume University. Male SHRs were purchased from SLC Inc. (Shizuoka, Japan) and housed under standard conditions of humidity, room temperature, and a 12-h:12-h dark-light cycle. They were provided with free access to tap water and chow.

\section{Study groups}

SHRs were randomly assigned into the following four groups $(n=20$ per group): Sham+vehicle, receiving sham operation and vehicle; Sham+ simvastatin, receiving sham operation and simvastatin; SAD+vehicle, receiving $\mathrm{SAD}$ and vehicle; and SAD+simvastatin, receiving SAD and simvastatin. At 12weeks-old, the SAD or sham operation was performed. In the SAD+simvastatin and Sham+simvastatin groups, $0.2 \mathrm{mg} \mathrm{kg}^{-1}$ per day simvastatin (Merck Research Laboratories, Rahway, NJ, USA) was intraperitoneally administered every day beginning at 7 days after the operation. This dose of simvastatin was the maximum dose that did not reduce BP in SHRs during the observation period. Before use, simvastatin was activated by alkaline hydrolysis to open the lactone rings, thereby converting it to its dihydroxy-open form or the activemetabolite form. ${ }^{20,21}$

\section{Bilateral SAD}

At 12 weeks old, the SHRs underwent bilateral SAD as previously described. ${ }^{13,22}$ Briefly, the rats were anesthetized intraperitoneally with a mixture of ketamine $\left(50 \mathrm{mg} \mathrm{kg}^{-1}\right)$, medetomidine $\left(0.5 \mathrm{mg} \mathrm{kg}^{-1}\right)$ and atropine sulfate $\left(0.5 \mathrm{mg} \mathrm{kg}^{-1}\right)$. The aortic depressor nerve and the superior laryngeal nerve were cut bilaterally. The bilateral superior cervical ganglia and cervical sympathetic trunks were resected. Thereafter, the carotid bifurcation and the external and internal carotid arteries were bilaterally stripped off the surrounding connective tissues, followed by the application of $10 \%$ phenol in absolute ethanol. The Sham-operated SHRs underwent bilateral isolation of the neck muscle alone.

\section{Telemetric hemodynamic recording and data analysis}

At 6 weeks after SAD, the rats underwent implantation of a BP radiotelemeter (model TA 11 PA-C 40, Data Sciences International, Minneapolis, MN, USA) under anesthesia with intraperitoneal pentobarbital $\left(30 \mathrm{mg} \mathrm{kg}^{-1}\right) \cdot{ }^{13}$ Thereafter, the rats were housed individually in a hemodynamic monitoring cage. At 7 days after telemeter implantation (19-weeks-old), 24-h BP and heart rate were monitored under the unrestricted, conscious condition. The 24-h average, standard deviation, and coefficients of the variance of mean BP and heart rate were calculated for descriptive statistics of the distribution variability. ${ }^{13}$

\section{Morphometric analysis and immunohistostaining}

After hemodynamic monitoring, the rats were killed with an intraperitoneal injection of overdose of pentobarbital. Blood was drawn from the right atrium

\section{A}
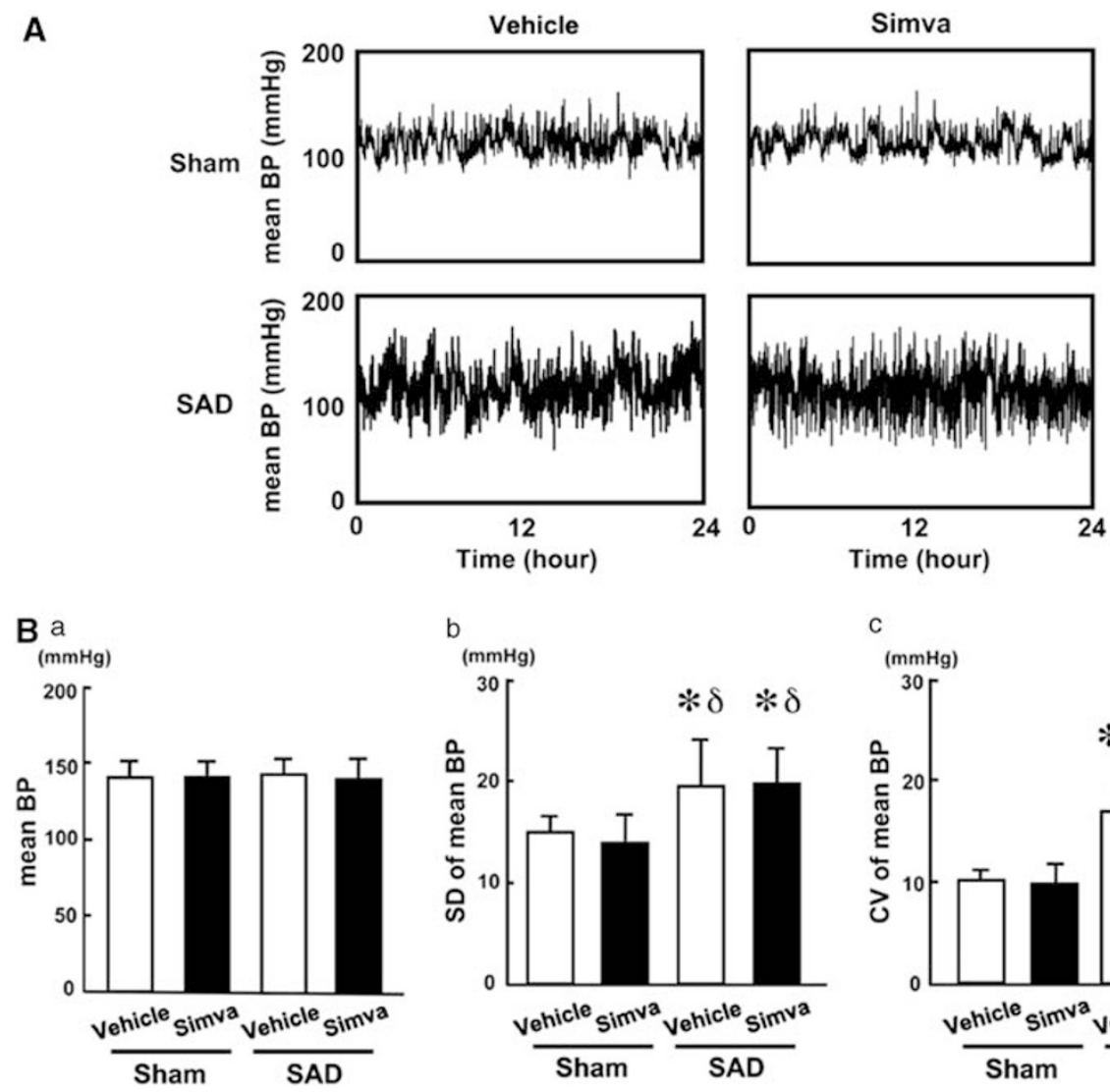

b

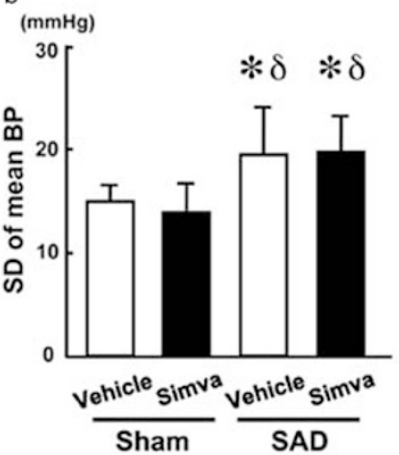

C

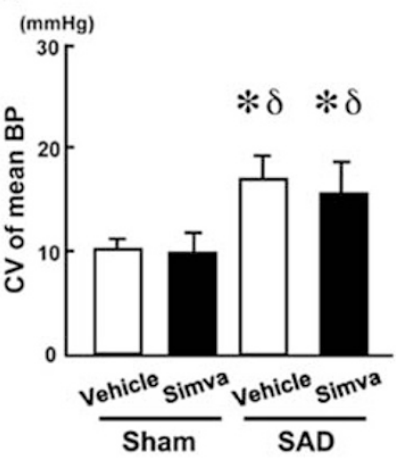

Figure 1 Effects of sinoaortic denervation (SAD) and simvastatin (Simva) on mean blood pressure (BP) and BP variability in spontaneously hypertensive rats (SHRs) at 7 weeks after the operation. A, Representative 24-h telemetric recordings of mean BP. B, Pooled data of the effects of SAD and simva on the average (a), standard deviation (s.d.) (b), and coefficient of variance (CV) (c), of mean BP in SHRs. Bar $=1 \times$ s.d. $(n=20)$. ${ }^{*} P<0.05$ vs. Sham+vehicle; ${ }^{\delta} P<0.05$ vs. Sham+Simva. 
to measure serum lipids using an automated analyzer (DRI-CHEM3500V, Fuji Film, Tokyo, Japan). After rats were perfuse fixed with $4 \%$ neutrally buffered paraformaldehyde at $100 \mathrm{~mm} \mathrm{Hg}$, the left ventricle (LV) was isolated from both atria and the right ventricular free wall, weighed, and immediately embedded in paraffin. The paraffinized sections ( $n=10$ /group) were subjected to morphometry and immunohistostaining. ${ }^{23}$ The shortest transverse myocyte diameter and the percent area of myocardial fibrosis, respectively, were measured in three independent hematoxylin/eosin-stained sections and Mallory-Azan-stained sections of each rat. ${ }^{23,24}$ Macrophages labeled with an antibody for ED-1 (Chemicon International, Temecula, CA, USA) were counted at $\times 200$ magnification in three independent entire cross-sections of each animal. ${ }^{25}$

\section{Echocardiographic studies}

Under anesthesia with intraperitoneal ketamine $\left(50 \mathrm{mg} \mathrm{kg}^{-1}\right)$ and xylazine $\left(10 \mathrm{mg} \mathrm{kg}^{-1}\right.$ ), echocardiographic measurements ( $n=10$ per group) of LV mass and LV fractional shortening were performed using a commercially available echocardiographic machine equipped with a 7.5-MHz transducer (SDD 5500, Aloca, Tokyo, Japan). ${ }^{23}$

\section{Real-time reverse-transcription PCR analysis}

Immediately after hemodynamic monitoring, the rats were killed with an intraperitoneal injection of an overdose of pentobarbital and then perfused

Table 1 Effects of SAD and simvastatin on heart rate and lipid profile

\begin{tabular}{|c|c|c|c|c|}
\hline & \multicolumn{2}{|c|}{ Sham } & \multicolumn{2}{|c|}{$S A D$} \\
\hline & Vehicle & Simvastatin & Vehicle & Simvastatin \\
\hline \multicolumn{5}{|l|}{ Heart rate (beats per min) } \\
\hline Average & $313 \pm 18$ & $322 \pm 11$ & $296 \pm 18$ & $304 \pm 18$ \\
\hline s.d. & $41 \pm 8$ & $40 \pm 4$ & $40 \pm 5$ & $38 \pm 5$ \\
\hline Coefficient of variance & $13 \pm 2$ & $12 \pm 1$ & $13 \pm 1$ & $12 \pm 2$ \\
\hline \multicolumn{5}{|l|}{ Lipid profile } \\
\hline Total cholesterol (mg per $100 \mathrm{ml}$ ) & $68 \pm 10$ & $69 \pm 6$ & $65 \pm 6$ & $64 \pm 5$ \\
\hline $\begin{array}{l}\text { High-density lipoprotein } \\
\text { cholesterol (mg per } 100 \mathrm{ml} \text { ) }\end{array}$ & $49 \pm 99$ & $45 \pm 5$ & $45 \pm 8$ & $44 \pm 8$ \\
\hline Triglycerides (mg per 100 ml) & $47 \pm 16$ & $47 \pm 112$ & $48 \pm 8$ & $46 \pm 12$ \\
\hline
\end{tabular}

Abbreviation: SAD, sinoaortic denervation

Data are mean \pm s.d. There were no differences among the four groups. with ice-cold saline for $5 \mathrm{~min}$. Unfixed LVs ( $n=10$ per group) were isolated, snap-frozen in liquid nitrogen, and stored at $-80{ }^{\circ} \mathrm{C}$ until use for real-time reverse-transcription-PCR analysis, immunoblotting and RhoA and Ras activity assays. Total RNA was extracted and reverse-transcribed as described previously. ${ }^{26}$ Equal amounts of the resulting cDNA were subjected to real-time PCR for rat brain natriuretic peptide (BNP), rat type 1 procollagen, rat transforming growth facto (TGF- $\beta$ ) and rat monocyte chemoattractant protein (MCP)-1. ${ }^{23,27}$ Expression levels of the target genes were normalized to GAPDH.

\section{RhoA and Ras activity assays}

Unfixed LV samples were homogenized in lysis buffer using FastPrep homogenizer (MP-Biomedicals, Irvine, CA, USA). RhoA-GTPase and Ras-GTPase levels were measured using the G-LISA RhoA activation assay kit (Cytoskeleton, Denver, CO, USA) and the Ras GTPase Chemi ELISA kit (Active Motif Japan, Tokyo, Japan), respectively.

\section{Immunoblotting}

After homogenization in the lysis buffer, total protein was extracted, separated by $10 \%$ sodium dodecyl sulfate-polyacrylamide gel electrophoresis, and subjected to immunoblotting. ${ }^{28,29}$ Blots were probed with an antibody against ERK1/2 or phosphorylated ERK1/2 (Cell Signaling Technology, Beverly, MA, USA), and the signals were detected with an enhanced chemiluminescence reagent kit (Amersham Pharmacia Biotech Inc. (Piscataway, NJ, USA). ${ }^{27,30}$ The intensity of immunoreactive bands was quantified by an image analysis system (ATTO, Tokyo, Japan).

\section{Statistical analysis}

Data are expressed as mean \pm s.d. Quantitative histological analysis was performed by two observers in a blinded manner. The interobserver or intraobserver variability was $<5 \%$ in each experiment. One-way ANOVA followed by Scheffe's F test was performed for the comparisons. A $P$ value of $<0.05$ was considered statistically significant.

\section{RESULTS}

Effects on mean BP and BP variability

Figure 1A shows representative 24-h telemetric recordings of mean BP at 7 weeks after SAD or sham operation (19-weeks-old). The mean BP levels were similar in the Sham+vehicle, Sham+simvastatin, $\mathrm{SAD}+$ vehicle and $\mathrm{SAD}+$ simvastatin groups (Figure 1B). SAD increased parameters of BP variability, namely, the standard deviation and coefficient of the variance of mean BP. Simvastatin did not affect
A

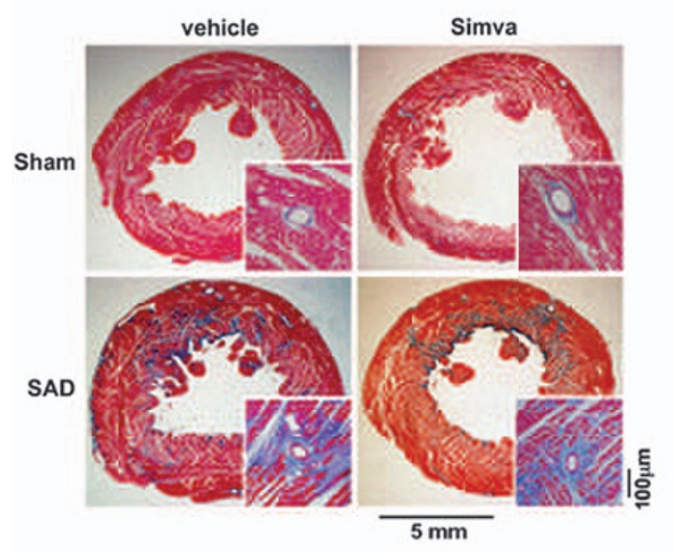

B a

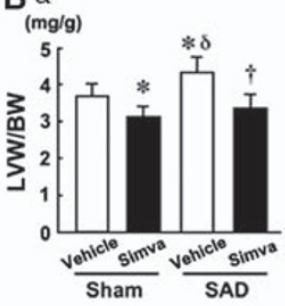

b

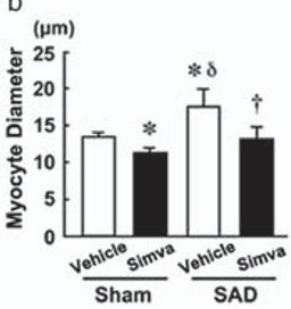

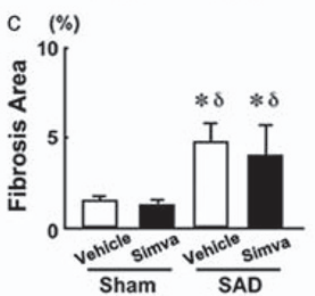

Figure 2 A, Representative photographs of Mallory-Azan stainings of the whole sections and microphotographs (inlets) of the left ventricles (LVs). Fibrotic tissue is stained blue. B, Pooled data of the effects of SAD and simva on LVW/BW (a), the minimum transverse myocyte diameter (b), and percent myocardial fibrosis area (c). Bar $=1 \times$ s.d. $(n=10)$. ${ }^{*} P<0.05$ vs. Sham+vehicle; ${ }^{\delta} P<0.05$ vs. Sham+Simva; ${ }^{\dagger} P<0.05$ vs. SAD+vehicle. 
the average or the variability of mean BP. The average and variability of heart rate did not differ among the four groups (Table 1). Simvastatin did not change the serum level of total cholesterol, high-density lipoprotein or triglycerides.

\section{Effects on cardiac hypertrophy}

$\mathrm{SAD}$ increased the ratio of $\mathrm{LV}$ wet weight to body weight (LVW/BW), myocyte diameter and BNP mRNA expression, which is a molecular marker of myocardial hypertrophy (Figures 2 and 3Aa). In the Sham+simvastatin group, LVW/BW, myocyte diameter and BNP expression were decreased as compared with the Sham+vehicle group, although the change in BNP expression did not reach statistical significance. In the $\mathrm{SAD}+$ simvastatin group, LVW/BW, myocyte diameter and BNP expression were reduced to levels not significantly different from those of the Sham+vehicle and Sham+simvastatin groups.

\section{Effects on myocardial fibrosis}

In the Sham+vehicle group, there was mild perivascular fibrosis (Figure 2). SAD not only enhanced perivascular fibrosis, but also induced patchy and massive reparative fibrosis, resulting in increased myocardial fibrosis area associated with upregulation of TGF- $\beta$ and type 1 procollagen mRNAs (Figures 2 and $3 \mathrm{Ab}-\mathrm{c}$ ). Simvastatin had little effect on myocardial fibrosis and the expression of TGF- $\beta$ and procollagen. SAD induced MCP-1 upregulation and macrophage infiltration (Figures 3Ad and B). Simvastatin did not affect MCP-1 expression or macrophage count.

\section{Effects on echocardiographic data}

SAD increased echocardiographic LV mass index (Figure 4a). Simvastatin prevented the SAD-induced aggravation of LV hypertrophy. In sham-operated SHRs, simvastatin reduced LV mass index, but the change was not significant. LV fractional shortening did not differ between the Sham+vehicle and Sham+simvastatin groups (Figure $4 \mathrm{~b}$ ). SAD reduced LV fractional shortening. Simvastatin slightly attenuated SAD-induced LV dysfunction, although this effect did not reach statistical significance.

\section{Effects on SAD-activated signaling pathways}

The activity levels of RhoA (not significant) and Ras $(P<0.01)$ were lower in the Sham+simvastatin group than in the Sham+vehicle group (Figure 5a). SAD increased RhoA and Ras activity levels. Simvastatin inhibited the SAD-induced activation of RhoA and Ras, reducing their activities to levels similar to those of Sham+simvastatin group. Next, we investigated the phosphorylation levels of ERK1/2, which is a Rasdependent mitogen-activated protein kinase. SAD increased ERK1/2 phosphorylation (Figure 5b), whereas total ERK1/2 levels did not differ among the four groups. The SAD-induced elevation of ERK1/2 phosphorylation was inhibited by simvastatin.

\section{DISCUSSION}

This study has shown that simvastatin ameliorates not only hypertensive hypertrophy in sham-operated SHRs, but also the large BP variability induced aggravation of hypertrophy in SAD-operated SHRs, whereas not changing the mean BP and BP variability. Simvastatin inhibited the SAD-induced activation of the RhoA/RasERK pathways in SHRs. In contrast, simvastatin had little effect on myocardial fibrosis in SAD-operated SHRs. Plasma lipid levels were also not affected by simvastatin.

The SAD procedure itself induces transient sympathetic nerve activation immediately after the operation, and it may lead to other, currently unknown changes. However, our recent study has
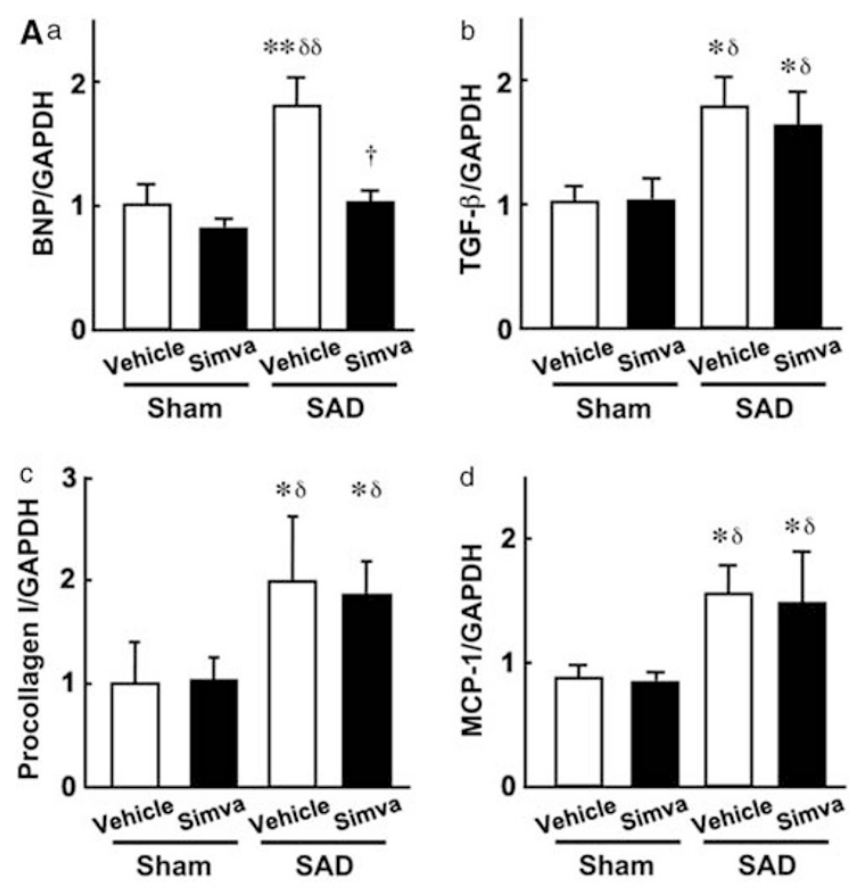

B

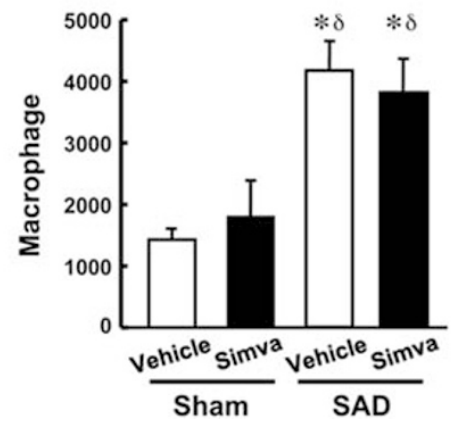

Figure 3 A, Pooled data of real-time reverse-transcription-PCR analysis showing the effects of $S A D$ and simva on myocardial brain natriuretic peptide (BNP) (a), transforming growth factor (TGF- $\beta$ ) (b), type I procollagen (c), and monocyte chemoattractant protein (MCP-1) (d) mRNA expression. Expression levels of target genes are normalized to GAPDH. Bar $=1 \times$ s.d. $(n=6)$. B, Pooled data of the effects on the ED-1-labeled macrophage count (per section). Bar $=1 \times$ s.d. $\quad(n=10) . \quad{ }^{*} P<0.05$ and ${ }^{* *} P<0.01$ vs. Sham+vehicle; ${ }^{\delta} P<0.05$ and $\delta \delta P<0.01$ vs. Sham+Simva; ${ }^{\dagger} P<0.05$ vs. SAD+vehicle.
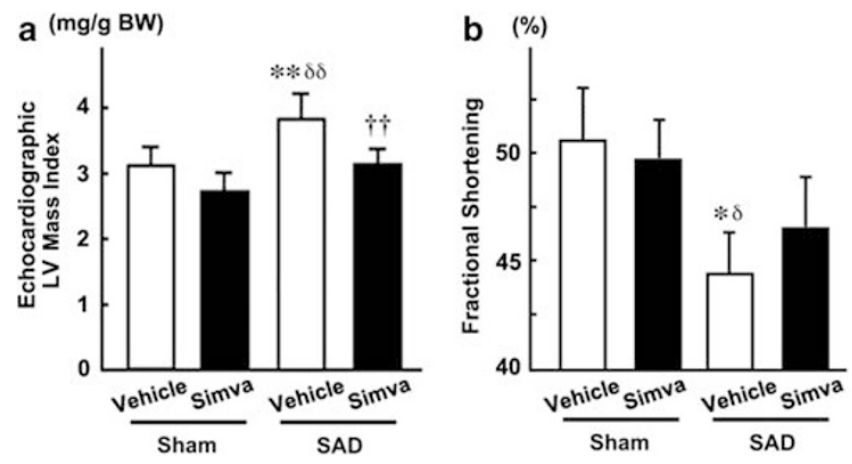

Figure 4 Pooled data showing the effects of simva on echocardiographic LV mass index (a) and LV fractional shortening (b). Bar=1×s.d. $(n=10)$. ${ }^{*} P<0.05$ and ${ }^{* *} P<0.01$ vs. Sham+vehicle; ${ }^{\delta} P<0.05$ and $\delta \delta P<0.01$ vs. Sham+Simva; ${ }^{\dagger} P<0.01$ vs. SAD+vehicle. 
a

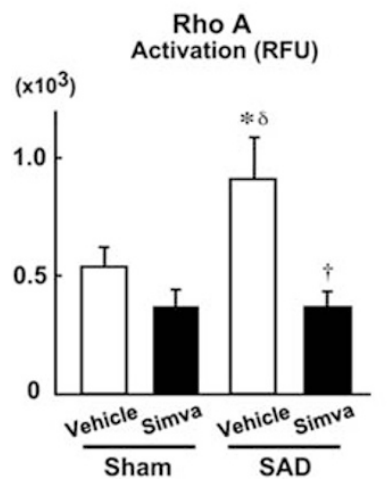

Ras

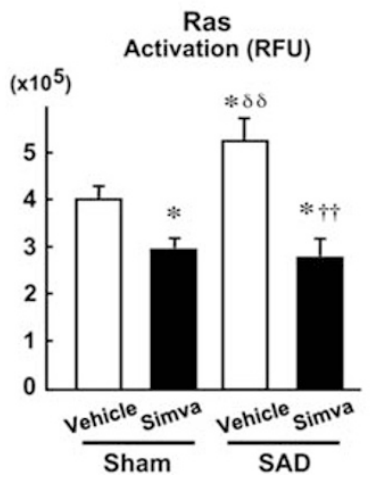

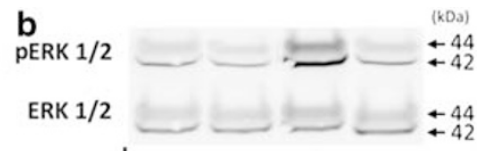

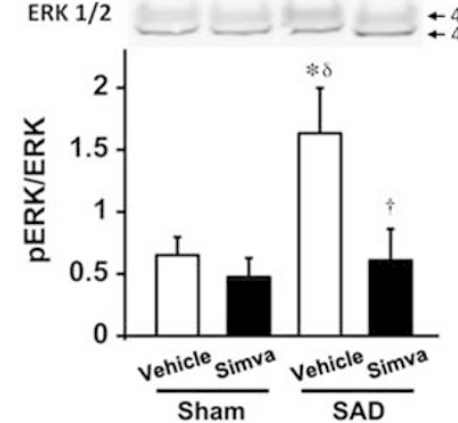

Figure 5 a, Effects of SAD and simva on RhoA (left) and Ras (right) activity levels. Relative fluorescence unit (RFU). Bar $=1 \times \operatorname{s.d}$. ( $n=10$ ). b, Effects of SAD and simva on ERK1/2 activity levels. Top, representative immunoblots for protein expression (ERK1/2) and phosphorylation level of ERK1/2 (pERK1/2). Bottom, pooled data of the effects on ERK phosphorylation levels. The density of the phosphorylated p42 band was normalized to that of p42 in each sample (pERK/ERK). Bar $=1 \times$ s.d. $(n=6)$. ${ }^{\star} P<0.05$ vs. Sham+vehicle; ${ }^{\delta} P<0.05$ and ${ }^{\delta \delta} P<0.01$ vs. Sham+Simva; ${ }^{\dagger} P<0.05$ and ${ }^{\dagger \dagger} P<0.01$ vs. SAD+vehicle.

demonstrated that circulating levels of interleukin-1 $\beta$, tumor necrosis factor- $\alpha$, norepinephrine and active renin are not increased in SHRs at 7 weeks after SAD. ${ }^{13}$ Thus, SAD-induced chronic cardiac remodeling may be independent of systemic inflammation, the systemic reninangiotensin system, and sympathetic nerve activation. It is likely that the effect of SAD is attributable to increased BP variability in the chronic phase of this model.

Simvastatin reduced cardiac hypertrophy without changing the mean BP in sham-operated SHRs. This finding is in agreement with earlier studies demonstrating that statins attenuate hypertensive cardiac hypertrophy in rats receiving chronic angiotensin II infusion or rats with thoracic aorta constriction. ${ }^{18,31}$ In this study, simvastatin inhibited the aggravation of cardiac hypertrophy in SHRs with large $\mathrm{BP}$ variability. These findings suggest that statins not only attenuate hypertrophic changes in response to static, chronic pressure overload (for example, SHRs and rats with aortic constriction) or neurohumoral stimuli (for example, rats with angiotensin II infusion), but also inhibit hypertrophy induced by dynamic, short-term BP changes (for example, increased BP variability).

The Ras-ERK1/2 pathway activates hypertrophic gene expression in various models of cardiac hypertrophy. ${ }^{32,33}$ RhoA is important for ERK1/2 activation in the mechanical stress-induced hypertrophy of cardiac myocytes. ${ }^{17,34}$ Thus, the RhoA/Ras-ERK1/2 pathways are implicated in the signaling system that has a role in cardiac hypertrophy in response to hypertension. Another important finding of this study was that simvastatin prevented the SAD-induced activation of RhoA, Ras and ERK1/2 in SHRs. Isoprenylation is essential for the activation of RhoA and Ras, as well as ERK1/2, in cardiac myocytes. ${ }^{35}$ Because inhibition of isoprenylation is one molecular mechanism underlying the pleiotropic effects of statins, ${ }^{15,16}$ our findings suggest that the RhoA/Ras-ERK pathway has a role in the aggravation of hypertensive cardiac hypertrophy induced by large BP variability.

Unexpectedly, simvastatin did not affect myocardial fibrosis and the expression of TGF- $\beta$ or procollagen in this model. Recently, we have shown that the SAD-induced aggravation of myocardial fibrosis depends on the MCP-1-mediated macrophage infiltration associated with TGF- $\beta$ induction. ${ }^{13}$ In this study, we demonstrated that SAD induced the aggravation of myocardial fibrosis associated with MCP-1 and TGF- $\beta$ upregulation and macrophage infiltration. We think that simvastatin did not decrease myocardial fibrosis in this model because simvastatin did not inhibit the SAD-induced upregulation of MCP-1,
TGF- $\beta$ or macrophage infiltration. In this regard, it is interesting to note that myocyte hypertrophy and myocardial fibrosis are regulated independently in hypertensive hearts. ${ }^{36,37}$ Mechanical stress itself has a role in initiating hypertrophic responses in myocytes by activating multiple signaling pathways. ${ }^{38,39}$ In contrast, the circulating and autocrine/paracrine humoral factors, such as angiotensin II, TGF- $\beta$ and MCP-1, have been implicated in hypertensive myocardial fibrosis. ${ }^{23,25,40-42}$ Further research is necessary, to determine the mechanism by which simvastatin induces the different effects between myocyte hypertrophy and myocardial fibrosis in this model.

In this study, simvastatin slightly attenuated SAD-induced LV systolic dysfunction, but the effect was not statistically significant. In contrast, our recent study has demonstrated that a subdepressor dose of candesartan, an angiotensin II type-1 receptor (AT1R) blocker, prevents LV dysfunction in SAD-operated SHRs. ${ }^{13}$ It is noteworthy that candesartan inhibits the aggravation of not only cardiac hypertrophy, but also perivascular and reparative fibrosis, although simvastatin does not affect myocardial fibrosis, in SAD-operated SHRs. These findings suggest that the lack of reduction of myocardial fibrosis by simvastatin may explain why simvastatin failed to prevent SAD-induced LV dysfunction.

There are several limitations to this study. First, the molecular mechanisms of RhoA and Ras-ERK1/2 activation were not clarified. During direct activation by mechanical stress, G protein-coupled receptor agonists, such as angiotensin II, endothelin-1 and phenylepherine, activate the Ras-ERK cascade via protein kinase $\mathrm{C}$ activation and subsequently upregulate hypertrophic gene upregulation in cardiac myocytes. ${ }^{32}$ In addition, angiotensin II and $\alpha$-adrenergic agonists activate RhoA through $\mathrm{G} \alpha_{\mathrm{q}} / \mathrm{G} \alpha_{11}$ proteins in cardiac myocytes. ${ }^{17,43} \mathrm{We}$ have demonstrated that the cardiac angiotensin II system is activated in SAD-operated SHRs. ${ }^{13}$ Therefore, angiotensin II may be a candidate that links large BP variability and RhoA/Ras-ERK activation in this model. Second, simvastatin prevents cardiac hypertrophy in rats receiving angiotensin II infusion. ${ }^{44}$ Statins downregulate AT1R expression in platelets and vascular smooth muscle cells. ${ }^{45,46}$ Thus, simvastatin may prevent SAD-induced aggravation of hypertensive cardiac hypertrophy, at least in part, by inhibiting the angiotensin II-mediated mechanism. This issue should be addressed in future studies. Additionally, pitavastatin prevents cardiac hypertrophy and myocardial fibrosis in Dahl salt-sensitive rats. ${ }^{47}$ Currently, the discrepancies between this and previous studies are not clear. The difference in 
statins used might be important because pitavastatin shows a 10-fold greater inhibitory effect on HMG-CoA reductase than that of simvastatin. ${ }^{48}$ We do not deny the possibility that larger doses of simvastatin would attenuate myocardial fibrosis in this model. Finally, several signaling pathways, such as p38 mitogen-activated protein kinase and p125 focal adhesion kinase, are implicated as mediators of stretchinduced myocyte hypertrophy. ${ }^{32}$ Thus, it remains to be elucidated whether such pathways would contribute to the SAD-induced aggravation of cardiac hypertrophy in SHRs.

In conclusion, simvastatin inhibited the aggravation of cardiac hypertrophy in a rat model of a combination of hypertension and large $\mathrm{BP}$ variability, without changing the mean $\mathrm{BP}$ and $\mathrm{BP}$ variability. Our findings suggest that the function of simvastatin in this model occurs via the inhibition of the RhoA/Ras-ERK1/2 pathways, independent of its lipid-lowering effect. Large BP variability is a characteristic feature of hypertensive patients with advanced atherosclerosis, especially with carotid atherosclerosis and in the elderly. Moreover, cardiac hypertrophy is an independent risk factor of cardiovascular events. However, there is no treatment currently available to directly reduce BP variability. This study raises the possibility that statins could be useful for preventing the exacerbation of cardiac hypertrophy in hypertensive patients with large BP variability.

\section{CONFLICT OF INTEREST}

The authors declare no conflict of interest.

\section{ACKNOWLEDGEMENTS}

We thank Kaoru Moriyama, Reiko Fujiyoshi, Michiko Taguchi, Miho Kogure, Miyuki Nishikata, Katsue Shiramizu and Kimiko Kimura for their skilful technical assistance. This study was supported in part by a grant for the Science Frontier Research Promotion Centers (Cardiovascular Research Institute); by Grants-In-Aid for Scientific Research (H Kai) from the Ministry of Education, Science, Sports and Culture, Japan; by a Research Grant for Cardiovascular Diseases from the Kimura Memorial Heart Foundation (H Kai); and by a Research Grant from the Ishibashi Memorial Foundation (H Kai).

1 Yano Y, Kario K. Unresolved issues of the morning blood pressure surge: the next stage of clinical applicability for the morning surge. Hypertens Res 2008; 31: 1491-1494.

2 Metoki H, Ohkubo T, Imai Y. Diurnal blood pressure variation and cardiovascular prognosis in a community-based study of Ohasama, Japan. Hypertens Res 2010; 33: 652-656.

3 Mancia G, Ferrari A, Gregorini L, Parati G, Pomidossi G, Bertinieri G, Grassi G, di Rienzo $M$, Pedotti A, Zanchetti A. Blood pressure and heart rate variabilities in normotensive and hypertensive human beings. Circ Res 1983; 53: 96-104.

4 Floras JS, Hassan MO, Jones JV, Osikowska BA, Sever PS, Sleight P. Factors influencing blood pressure and heart rate variability in hypertensive humans. Hypertension 1988; 11: $273-281$.

5 Canonico V, De Caprio L, Vigorito C, Forgione L, Tedeschi C, Guarini P, Rengo F. Differences in blood pressure profile between young and elderly hypertensive patients. J Hum Hypertens 1990; 4: 405-409.

6 Zakopoulos NA, Tsivgoulis G, Barlas G, Papamichael C, Spengos K, Manios E, Ikonomidis I, Kotsis V, Spiliopoulou I, Vemmos K, Mavrikakis M, Moulopoulos SD. Time rate of blood pressure variation is associated with increased common carotid artery intima-media thickness. Hypertension 2005; 45: 505-512.

7 Parati G, Pomidossi G, Albini F, Malaspina D, Mancia G. Relationship of 24-h blood pressure mean and variability to severity of target-organ damage in hypertension. $J$ Hypertens 1987; 5: 93-98.

8 Sega R, Corrao G, Bombelli M, Beltrame L, Facchetti R, Grassi G, Ferrario M, Mancia G. Blood pressure variability and organ damage in a general population: Results from the PAMELA study (Pressioni Arteriose Monitorate E Loro Associazioni). Hypertension 2002; 39: 710-714.

9 Tatasciore A, Zimarino M, Renda G, Zurro M, Soccio M, Prontera C, Emdin M, Flacco M, Schillaci G, De Caterina R. Awake blood pressure variability, inflammatory markers and target organ damage in newly diagnosed hypertension. Hypertens Res 2009; 31: 2137-2146.

10 Kikuya M, Hozawa A, Ohokubo T, Tsuji I, Michimata M, Matsubara M, Ota M, Nagai K, Araki T, Satoh H, Ito S, Hisamichi S, Imai Y. Prognostic significance of blood pressure and heart rate variabilities: The Ohasama study. Hypertension 2000; 36: 901-906.
11 Pringle E, Phillips C, Thijs L, Davidson C, Staessen JA, de Leeuw PW, Jaaskivi M, Nachev C, Parati G, O'Brien ET, Tuomilehto J, Webster J, Bulpitt CJ, Fagard RH. Systolic blood pressure variability as a risk factor for stroke and cardiovascular mortality in the elderly hypertensive population. J Hypertension 2003; 21: 2251-2257.

12 Eto M, Toba K, Akishita M, Kozaki K, Watanabe T, Kim S, Hashimoto M, Ako J, Iijima K, Sudoh N, Yoshizumi M, Ouchi Y. Impact of blood pressure variability on cardiovascular events in elderly patients with hypertension. Hypertens Res 2005; 28: 1-7.

13 Kudo H, Kai H, Kajimoto H, Koga M, Takayama N, Mori T, Ikeda A, Yasuoka S, Angawa $\mathrm{T}$, Mifune $\mathrm{H}$, Kato $\mathrm{S}$, Hirooka $\mathrm{H}$, Imaizumi T. Exaggerated blood pressure variability superimposed on hypertension aggravates cardiac remodeling in rats via angiotensin II system-mediated chronic inflammation. Hypertension 2009; 54: 832-838.

14 Kai H, Kudo H, Takayama N, Yasuoka S, Kajimoto H, imaizumi T. Large blood pressure variability and hypertensive cardiac remodeling -Role of cardiac inflammation-. Circ J 2009; 73: 2198-2203.

15 Liao JK. Statin therapy for cardiac hypertrophy and heart failure. J Investig Med 2004; 52: 248-253.

16 Ramasubbu K, Estep J, White DL, Deswal A, Mann DL. Experimental and clinical basis for the use of statins in patients with ischemic and nonischemic cardiomyopathy. J Am Coll Cardiol 2008; 51: 415-426.

17 Sah VP, Hoshijima M, Chien KR, Brown JH. Rho is required for Galphaq and alpha1adrenergic receptor signaling in cardiomyocytes. Dissociation of Ras and Rho pathways. J Biol Chem 1996; 271: 31185-31190.

18 Takemoto M, Node K, Nakagami H, Liao Y, Grimm M, Takemoto Y, Kitakaze M, Liao JK. Statins as antioxidant therapy for preventing cardiac myocyte hypertrophy. J Clin Invest 2001; 108: 1429-1437.

19 Higashi M, Shimokawa H, Hattori T, Hiroki J, Mukai Y, Morikawa K, Ichiki T, Takahashi $\mathrm{S}$, Takeshita A. Long-term inhibition of Rho-kinase suppresses angiotensin II-induced cardiovascular hypertrophy in rats in vivo: Effect on endothelial $\mathrm{NAD}(\mathrm{P}) \mathrm{H}$ oxidase system. Circ Res 2003; 93: 767-775.

20 Hamelin BA, Turgeon J. Hydrophilicity/lipophilicity: relevance for the pharmacology and clinical effects of HMG-CoA reductase inhibitors. Trends Pharmacol Sci 1998; 19: 26-37.

21 Girgis RE, Li D, Zhan X, Garcia JG, Tuder RM, Hassoun PM, Johns RA. Attenuation of chronic hypoxic pulmonary hypertension by simvastatin. Am J Physiol Heart Circ Physiol 2003; 285: H938-H945.

22 Krieger EM. Neurogenic hypertension in rat. Circ Res 1964; 15: 511-521.

23 Kuwahara F, Kai H, Tokuda K, Kai M, Takeshita A, Egashira K, Imaizumi T. Transforming growth factor- $\beta$ function blocking prevents myocardial fibrosis and diastolic dysfunction in pressure-overloaded rats. Circulation 2002; 106: 130-135.

24 Kai H, Muraishi A, Sugiu Y, Nishi H, Seki Y, Kuwahara F, Kimura A, Kato H, Imaizumi T. Expression of proto-oncogenes and gene mutation of sarcomeric proteins in patients with hypertrophic cardiomyopathy. Circ Res 1998; 83: 594-601.

25 Tokuda K, Kai H, Kuwahara F, Yasukawa H, Tahara N, Kudo H, Takemiya K, Koga M, Yamamoto T, Imaizumi T. Pressure-independent effects of angiotensin II on hypertensive myocardial fibrosis. Hypertension 2004; 43: 499-503.

26 Kusaba K, Kai H, Koga M, Takayama N, Ikeda A, Yasukawa H, Seki Y, Egashira K, Imaizumi T. Inhibition of intrinsic interferon- $\gamma$ function prevents neointima formation after balloon injury. Hypertension 2007; 49: 909-915.

27 Koga M, Kai H, Yasukawa H, Yamamoto T, Kawai Y, Kato S, Kusaba K, Kai M, Egashira $\mathrm{K}$, Kataoka $\mathrm{Y}$, Imaizumi T. Inhibition of progression and stabilization of plaques by therapeutic interferon- $\gamma$ function blocking in ApoE-KO mice. Circ Res 2007; 101: 348-356.

28 Kai H, Griendling KK, Lassegue B, Ollerenshaw JD, Runge MS, Alexander RW. Agonistinduced phosphorylation of the vascular type 1 angiotensin II receptors. Hypertension 1994; 24: 523-527.

29 Tahara N, Kai H, Niiyama H, Mori T, Sugi Y, Takayama N, Yasukawa H, Numaguchi Y, Matsui H, Okamura K, Imaizumi T. Repeated gene transfers of naked prostacyclin synthase plasmid into skeletal muscles attenuate monocrotaline-induced pulmonary hypertension and prolong survival in rats. Human Gene Ther 2004; 15: 1270-1278.

30 Kai H, Griendling KK, Lassègue B, Fukui T, Minieri CA, Alexander RW. Prolonged exposure to agonist results in a reduction in the levels of the $G_{q} / G_{11} \alpha$-subunit in cultured vascular smooth muscle cells. Mol Pharmacol 1996; 49: 96-104.

31 Indolfi C, Di Lorenzo E, Perrino C, Stingone AM, Curcio A, Torella D, Cittadini A, Cardone L, Coppola C, Cavuto L, Arcucci O, Sacca L, Avvedimento EV, Chiariello M. Hydroxymethylglutaryl coenzyme A reductase inhibitor simvastatin prevents cardiac hypertrophy induced by pressure overload and inhibits p21 ras activation. Circulation 2002; 106: 2118-2124.

32 Sugden PH. Signaling in myocardial hypertrophy: Life after calcineurin? Circ Res 1999; 84: 633-646.

33 Brown JH, Del Re DP, Sussman MA. The Rac and Rho hall of frame: A decade of hypertrophic signaling hits. Circ Res 2006; 98: 730-742.

34 Aikawa R, Komuro I, Yamazaki T, Zou Y, Kudoh S, Zhu W, Kadowaki T, Yazaki Y. Rho family small $G$ proteins play critical roles in mechanical stress-induced hypertrophic responses in cardiac myocytes. Circ Res 1999; 84: 458-466.

35 Thorburn J, Xu S, Thorburn A. MAP kinase- and Rho-dependent signals interact to regulate gene expression but not actin morphology in cardiac muscle cells. EMBO J 1997; 16: 1888-1900.

36 Weber KT, Brilla CG, Janicki JS. Myocardial fibrosis: Functional significance and regulatory factors. Cardiovasc Res 1993; 27: 341-348.

37 Nicoletti A, Michel JB. Cardiac fibrosis and inflammation: Interaction with hemodynamic and hormonal factors. Cardiovasc Res 1999; 41: 532-543.

38 Sadoshima J, Izumi S. The cellular and molecular response of cardiac mypcytes to mechanical stress. Annu Rev Physiol 1997; 59: 551-571. 
39 Harada K, Komuro I, Shiojima I, Hayashi D, Kudoh S, Mizuno T, Kijima K, Matsubara $\mathrm{H}$, Sugaya T, Murakami K, Yazaki Y. Pressure overload induces cardiac hypertrophy in angiotensin II type 1A receptor knockout mice. Circulation 1999; 97: 1952-1959.

40 Brilla CG, Weber KT. Reactive and reparative myocardial fibrosis in arterial hypertension in the rats. Cardiovasc Res 1992; 26: 671-677.

41 Nicoletti A, Heudes D, Mandet C, Hinglais N, Bariety J, Michel JB. Inflammatory cells and myocardial fibrosis: Spatial and temporal distribution in renovascular hypertensive rats. Cardiovasc Res 1996; 32: 1096-1107.

42 Kuwahara F, Kai H, Tokuda K, Takeya M, Takeshita A, Egashira K, Imaizumi T. Hypertensive myocardial fibrosis and diastolic dysfunction -Another model of inflammation-. Hypertension 2004; 43: 739-745.

43 Aoki H, Izumo S, Sadoshima J. Angiotensin II activates RhoA in cardiac myocytes: A critical role of RhoA in angiotensin II-induced premyofibril formation. Circ Res 1998; 82: 666-676.

44 Delbosc S, Cristol JP, Descomps B, Mimran A, Jover B. Simvastatin prevents angiotensin II-induced cardiac alteration and oxidative stress. Hypertension 2002; 40: 142-147.
45 Nickenig G, Bäumer AT, Temur Y, Kebben D, Jockenhövel F, Böhm M. Statin-sensitive dysregulated AT1 receptor function and density in hypercholesterolemic men. Circulation 1999; 100: 2131-2141.

46 Wassmann S, Laufs U, Bäumer AT, Müller K, Konkol C, Sauer H, Böhm M, Nickenig G. Inhibition of geranylgeranylation reduces angiotensin II-mediated free radical production in vascular smooth muscle cells: Involvement of angiotensin AT1 receptor expression and Rac1 GTPase. Mol Pharmacol 2001; 59: 646-654.

47 Saka M, Obata K, Ichihara S, Cheng XW, Kimata H, Noda A, Izawa H, Nagata K, Yokota M. Attenuation of ventricular hypertrophy and fibrosis in rats by pitavastatin: Potential role of the RhoA-extracellular signal-regulated kinase-serum response factor signalling pathway. Clin Exp Pharmacol Physiol 2006; 33: 1164-1171.

48 Aoki T, Nishimura H, Nakagawa S, Kojima J, Suzuki H, Tamaki T, Wada Y, Yokoo N, Sato F, Kimata H, Kitahara M, Toyoda K, Sakashita M, Saito Y. Pharmacological profile of a novel synthetic inhibitor of 3-hydroxy-3-methylglutaryl-coenzyme A reductase. Arzneimittelforschung 1997; 47: 904-909. 